\title{
Diz çevresi osteotomilerinde modern endikasyonlar, kontrendikasyonlar ve hasta seçimi
}

\section{Modern indications and contraindications of osteotomies around the knee and patient selection}

\author{
Onur Gürsan¹, Ahmet Emrah Açan², Hasan Tatari ${ }^{1}$ \\ ${ }^{1}$ Dokuz Eylül Üniversitesi Tıp Fakültesi Hastanesi, Ortopedi ve Travmatoloji Anabilim Dalı, İzmir \\ ${ }^{2}$ Balıkesir Üniversitesi Tıp Fakültesi Hastanesi, Ortopedi ve Travmatoloji Anabilim Dalı, Balıkesir
}

\begin{abstract}
Diz çevresinin düzeltici osteotomileri, instabilite eşlik etsin veya etmesin, frontal ve/veya sagittal planlardaki dizilim kusurlarını ortadan kaldırmak ve diz osteoartritini yönetmek amaçlı yenilikçi ve etkili terapötik (tedavi edici) yöntemlerdir. Başarı; öykü, fizik muayene ve tam radyolojik ölçümleri içeren ameliyat öncesi doğru hasta değerlendirmesine ve titiz planlamaya bağlıdır. Bu derleme, diz çevresi osteotomilerinde, doğru hasta seçimi, geçerli endikasyonlar ve kontrendikasyonları içerecek şekilde, güncel klinik bilgiler sunmaktadır. Daha önceleri kesin kontrendikasyonlar içerisinde yer alan dize ait bazı özel durumlar, yeni yöntemlerin gelişmesi ve popülasyonun değişen ihtiyaçlarına göre artık, göreceli kontrendikasyonlar grubu içerisinde yer almaktadır. Eklem kıkırdağına yönelik kıkırdak nakli gibi koruyucu işlemlerden sonra, varsa alt ekstremitedeki deformitenin düzeltilmesi, kıkırdak koruyucu yöntemlerin başarısını arttırmakta olup, rutin uygulamada giderek yaygınlaşmaktadır. Bu durum, diz çevresi osteotomilerinin uygulama alanlarını genişlettiği gibi, uygun hasta ve ideal cerrahi yöntem seçimi açısından daha ayrıntılı bir değerlendirme gerektirmektedir. Sonuç olarak, ideal kriterlere uygun endikasyonlar içerisinde osteotomi yapılması ve kontrendikasyonların göz önünde bulundurulması tatmin edici sonuçlara ulaşmada yardımcı olmaktadır.
\end{abstract}

Anahtar sözcükler: yüksek tibial osteotomi (YTO); distal

femoral osteotomi (DFO); eklem koruyucu cerrahi; hasta seçimi

\section{DiZ ÇEVRESI OSTEOTOMILER}

Osteotomi, instabilite eşlik etsin veya etmesin, frontal ve/veya sagittal plan deformitelerini tedavi etmek amacıyla, diz eklemini koruyucu cerrahi yöntem olarak bilinir. Bu yöntemde temel amaç; frontal ve/veya sagittal plan deformitelerini düzeltmek, yük dağılımını değiştirmek suretiyle eklem kıkırdağını korumak, koronal ve anteroposterior diz stabilizasyonunu optimize etmektir. ${ }^{[1]}$
Corrective osteotomies around knee are innovative and effective therapeutic methods for eliminating frontal and/or sagittal malalignment with or without instability and managing knee osteoarthritis. Successful outcome depends on accurate patient assessment and rigorous planning, including history, physical examination, and complete radiological evaluation. This review provides up-dated clinical information about the osteotomies around the knee, including appropriate patient selection, current indications and contraindications. Some specific conditions of knee joint, which were previously in definite contraindications, are now included in relative contraindications group according to development of new methods and the changing needs of the population. After conservative procedures such as cartilage transplantation for joint cartilage, if any deformity exists, correction of malalignment, increases the success of cartilage-preserving methods and is becoming increasingly common in routine practice. This not only extends the range of application of osteotomies around the knee, but also requires a more detailed assessment of appropriate patient and ideal surgical method selection. In conclusion, performing osteotomy within ideal indications and considering the contraindications help to achieve satisfactory outcome.

Key words: high tibial osteotomy (HTO); distal femoral osteotomy (DFO); joint preservative surgery; patient selection

Proksimal (yüksek) tibial osteotomi, yapısal veya travmatik sebeplerle oluşan metafizyal deformitelerin sonucu olarak, semptomatik varus dizlerin tedavisinde ideal bir seçenek olarak düşünülmektedir. ${ }^{[2]}$ Distal femoral osteotomiler, lateral kompartmanda aşırı yüklenmeyle birliktelik gösteren valgus dizlere çözüm olarak sunulmaktadır. ${ }^{[3]}$ Valgus deformitesinin, lateral menisektomi veya travma sonrası sebeplerle tibial kökenli olduğu durumlarda, proksimal tibia varus

- İletişim adresi: Uzm. Dr. Onur Gürsan, Dokuz Eylül Üniversitesi Tıp Fakültesi Hastanesi Ortopedi ve Travmatoloji Anabilim Dalı, İzmir Tel: 0505 - 2679819 e-posta: onur_84_gursan@hotmail.com

- Geliștarihi: 20 Ocak $2020 \quad$ Kabul tarihi: 7 Mart 2020 
osteotomisi bir çözüm olarak görülmektedir. ${ }^{[4]}$ Sagittal planda kemik kökenli dengesizliklerden kaynaklanan diz instabilitesi, sadece sagittal planda veya frontal planla birlikte yapılan kombine osteotomilerle tedavi edilen, az bilinen bir durumdur. ${ }^{[5]}$

Osteotominin amacı deformiteyi düzeltmek ve eklemin maruz kaldığı yüklerin yeniden dağılımını sağlamaktır. Koronal dizilim kusurları, kıkırdak sorunlarına yol açan medial veya lateral aşırı yüklenme durumlarıyla ilişkilidir. Medial veya lateral eklem aralığının daralmasıyla kıkırdak sorunları daha da artacağından, bu kısır döngünün çözümünde osteotomi kullanışlı bir seçenektir. ${ }^{[6,7]}$ Ancak, osteotomi endikasyonları arasında sadece koronal plan deformitelerinin düzeltilmesi bulunmaz. Sagittal plandaki dengesizlikler kemik kaynaklı veya bağ yetmezliği ile ilişkili olabilir. Artmış tibial eğim, ön çapraz bağda artmış gerilim ile ilişkilidir. Tibia posterior eğim açısını (slope) değiştirmek suretiyle sagittal plan düzeltmeleri de son zamanlarda ilgi görmektedir. Kronik ön çapraz bağ yaralanması ile ilişkili varus dizilim kusuru, kronik posterolateral köşe ve kronik arka çapraz bağ yaralanması ile birliktelik gösteren ön çapraz bağ yetmezliği ile ilişkili varus dizilim kusurlarında (ikili varus, rekurvatumun eşlik etmesiyle üçlü varus olarak tarif edilir) sagittal plan değişiklikleri yapılır. ${ }^{[8-10]}$

Kıkırdak otogreft veya allogreft transplantasyonlarında veya meniskal allogreft transplantasyonlarında, koronal plandaki deformiteler düzeltilmeden işlem yapıldığında, klinik olarak daha kötü sonuçlar bildirilmiştir. ${ }^{[11]}$

Tüm bu bilgiler çerçevesinde, diz çevresi osteotomilerinin genel endikasyonlarını özetleyecek olursak:

- Dizilim kusuru ve artrit

- Dizilim kusuru ve instabilite

- Dizilim kusuru, artrit ve instabilite

- Kondral veya meniskal transplantasyona eşlik eden dizilim kusuru olarak sıralama yapılabilir.[7]

\section{KORONAL PLANDAKI OSTEOTOMILER}

Klinik olarak; tek tibiofemoral kompartmanda sınırlı diz ağrısı, redükte edilemeyen deformite ve tam veya tama yakın eklem hareket açıklığı $\left(5^{\circ}-10^{\circ}\right.$ kontraktür, seçili vakalarda tolere edilebilir), radyolojik olarak; eklem aralığında daralma ve $5^{\circ}$ 'den fazla ekstra-artiküler (eklem dışı) deformite varlığı, koronal plandaki osteotomiler için ideal endikasyonlardır. Genel olarak, kemik ilişkili deformitenin bulunmaması (kontraktürler), inflamatuar artrit ve birden fazla kompartmanın tutulması, koronal plandaki osteotomiler için kontrendikasyon teşkil etmektedir. ${ }^{[6]}$

\section{Yüksek (Proksimal) Tibial Osteotomi}

Varus dizlerde, yüksek tibial osteotominin (YTO) endikasyonları ve kontrendikasyonları kesin sınırlarla belirlenmemiş olsa da, YTO adayı hasta tanımı yapılabilmektedir. Uluslararası Artroskopi, Diz Cerrahisi ve Ortopedik Spor Hekimliği Topluluğuna göre (ISAKOSInternational Society of Arthroscopy, Knee Surgery and Orthopaedic Sports Medicine) YTO adayı hasta; diz ekleminin medialinde lokalize ağrı bulunan ve basarak çekilen direkt grafide, medial artrozla ve varusla ilişkili olarak, medial eklem aralığında daralma saptanan, normal lateral ve patellofemoral kompartmana sahip, VKi (Vücut Kitle İndeksi) $30 \mathrm{~kg} / \mathrm{m}^{2}$ nin altında olan, koşma ve sıçrama dışında yüksek aktivite beklentisi olan, 40-60 yaş arası hasta olarak tanımlanabilir. ${ }^{[12]}$ Bununla birlikte, kıkırdak koruyucu cerrahi girişimlerde, hasarlı bölgeye binen yükün azalmasını sağlamak amacıyla yapılan osteotomilerde de endikasyon açısından görüş birliği mevcuttur. ${ }^{[13]}$

Yüksek (proksimal) tibial osteotomi için farklı görüşlerin olduğu endikasyonlar ve kontrendikasyonlar bulunmaktadır. ${ }^{[14]}$ Literatüre bakıldığında, ideal varus miktarı $10^{\circ} \mathrm{kabul}$ edilmekle birlikte, $15^{\circ}$ altını endikasyon içerisinde gören çalışmalar da dikkat çekmektedir. Puddu, $20^{\circ}$ üzerindeki varus değerlerini, düzeltmeyle birlikte instabiliteye yol açacağından kontrendike olarak kabul etmektedir. ${ }^{[3]}$ YTO başarısında en önemli etkenlerden biri operasyon öncesi eklem hareket açıklığıdır. Minimum $120^{\circ}$ diz eklem hareket açıklığı ve maksimum $5^{\circ}$ fleksiyon kontraktürü bulunan olgularda daha başarılı sonuçlar elde edilmiştir. ${ }^{[15,16]}$ Ahlback Evre I-Il tek kompartmantal artrozlarda endikasyon belirtilirken, daha ileri evrelerde yapılmış osteotomilere ait çalışmalar bulunmaktadır. Yapılan cerrahi ile medialdeki yükü, daha sağlıklı olduğunu düşündügümüz lateral kompartmana yönlendirmiş olsak dahi, Ahlback Evre III-IV hastalarda sonuçların daha başarısız olacağını tahmin etmek zor değildir. ${ }^{[17]}$ YTO'da patellar yüksekliğin azalması kabul edilen bir durumdur. Bu değişiklik, retropatellar temas basıncında artışa ve bunun sonucunda diz önü ağrılara yol açabilir. Ileri evre patellofemoral artrozu bulunan olguların, yüksek tibial osteotomi sonrasında başarısız sonuçlarla birliktelik göstermesi kaçınılmazdır. Patellofemoral artrozla birlikte yapılan osteotomi ameliyatlarında Maquet ve Fulkerson gibi diz önü yakınmalarının azalmasına yönelik yöntemler belirtilmiştir. ${ }^{[18-20]}$ ilk zamanlarda patella baja varlığı kontrendikasyon olarak kabul edilmekteydi. Lobenhoffer ve ark. her $10^{\circ}$ 'lik valgus düzeltmesine karşııı patella yüksekliğini $2 \mathrm{~mm}$ azaltan biplanar osteotomiyi tarif ettiler. ${ }^{[21]}$ Patella baja varlığında uniplanar kapalı kama osteotomisi patella yüksekliğini artırabilirken, biplanar osteotomiler patella yüksekliğinin 
değişmeden kalmasını sağlayabilmektedir. Patella alta varlığında, hem biplanar proksimal frontal açık kama osteotomisi hem de uniplanar açık kama osteotomi patella altayı azaltma etkisine sahiptir. ${ }^{[22-24]}$

Yukarıda belirtildiği gibi, varolan dizilim kusuru düzeltilmediğinde, uygulanan mikrofraktür veya osteokondral transplantasyonun başarısız olduğunun (\%56) fark edilmesinden bu yana, yüksek tibial osteotomi, kıkırdak rekonstrüksiyon işlemlerine ek olarak uygulanmaktadır. ${ }^{[25,26]}$ Femur medial kondilinde fokal kıkırdak lezyonlarına eşlik eden, $5^{\circ}$ 'den fazla varus varlığında, genel yaklaşım eş zamanlı ya da iki aşamalı olarak deformitenin de düzeltilmesi yönündedir. ${ }^{[13]}$ Randomize olmayan, kontrol gruplu klinik çalışmaya göre, medial femoral kondilde kıkırdak defektlerine otolog kondrosit implantasyonu uygulanan $5^{\circ}$ 'den az varusu olan hastaların, yüksek tibial osteotomisiz ve osteotomili sonuçlarına bakıldığında; YTO uygulanan grupta daha başarılı sonuçlar görülmüştür. ${ }^{[27]}$ Bu nedenle, kıkırdak rekonstrüksiyon işlemlerinde deformiteyi değerlendirmek gerektiği düşüncesindeyiz.

YTO için üst yaş sınırı 65 yaş olarak bildirilmesine rağmen, diğer koşulları sağlayan olgularda daha ileri yaşlarda osteotomi yapılan serilere rastlanılmaktadır. ${ }^{[28]} \mathrm{Bu}$ nedenle, 65 yaş üzeri hastalar rölatif (göreceli) kontrendikasyon grubuna dahil edilmektedir. Diğer rölatif kontrendikasyonlar içerisinde, obezite (VKI >30 $\mathrm{kg} / \mathrm{m}^{2}$ ), patella infera veya alta olması ve ciddi patellofemoral semptomların varlığı sayılabilir. Özetleyecek olursak;

\section{YTO endikasyonları:}

- Sedanter olmayan uyumlu hasta

- 65 yaş altı hasta

- $5^{\circ}-15^{\circ}$ varus dizilimi

- $90^{\circ}-120^{\circ}$ aktif eklem hareket açıklığı

- Hareketle artan bölgesel ağrı

- $5^{\circ}$ 'den az fleksiyon kontraktürü

- Ahlback Evre I-Il tek kompartmantal artroz

\section{YTO kesin kontrendikasyonları:}

- Diffüz dejeneratif artrit

- Inflamatuar artrit

- Tibiofemoral subluksasyon

- Ileri derece osteoporoz

- $90^{\circ}$ 'den az eklem hareket açıklığı
- $15^{\circ}$ 'den fazla varus

- Ciddi vasküler patoloji

- Lateral menisektomi öyküsü

- Gerçek dışı hasta beklentisi

\section{YTO rölatif (göreceli) kontrendikasyonları:}

- Ciddi patellofemoral bulguların olması

- Patella alta veya infera olması

- Hastanın 65 yaş üzerinde olması

- Obezite $\left(\mathrm{VKI}>30 \mathrm{~kg} / \mathrm{m}^{2}\right)$ şeklinde değerlendirilebilir.

\section{Distal Femoral Osteotomi}

Alt ekstremite anatomik aksı ile mekanik aksı arasında $10^{\circ}-15^{\circ}$ valgusu bulunan ve lateral kompartman üzerinde lokalize ağrısı olan hastalarda, distal femoral osteotomiler kullanışlı olabilmektedir. Distal femoral osteotominin kontrendikasyonları arasında, eş zamanlı olarak tedavi edilmedikçe, ligamentöz instabilite varlığı, şiddetli trikompartmantal artrit varlığı, lateral kondilin osteonekrozu, inflamatuar artrit ve tibianın subluksasyonu ile birliktelik gösteren $20^{\circ}$ 'den fazla valgus deformitesi vardır. ${ }^{[29]}$

\section{SAGITTAL PLANDAKI OSTEOTOMILER}

Sagittal düzlem deformitesi ile ilgili olarak, güncel literatür, ön çapraz bağ revizyon cerrahisi geçiren olgularda ve posterior tibial eğim açısının $12^{\circ}$ 'den fazla olduğu durumlarda, tibial anterior kapalı kama osteotomisini (tibial dorsifleksiyon osteotomisi) önermektedir. Tibianın anterior açık kama osteotomisi (antekurvatum, tibial fleksiyon osteotomisi) inverte posterior tibial eğim açısıyla ve/veya ligaman yetmezlikleriyle birliktelik gösteren semptomatik (ağrı, instabilite) genu rekurvatumda endikedir. Rekurvatum deformitesi, kemik deformitesi olmadan kapsüloligamentöz travmatik yetmezliğe ikincil ise, rekonstrükte edilmiş ligamentleri koruyabilmesine rağmen, tibial fleksiyon osteotomisi için daha az gerekçe vardır. ${ }^{[30]}$

Genel olarak, bir osteotomi göz önüne alındığında, klinik ve radyolojik olmayan, ancak temel olan bir diğer kriter, hasta uyumudur. Osteotomi, fonksiyonu tamamen korumak için değişen aralıklarda tam yük vermeden veya kontrollü yüklenme ile mobilizasyon gerektiren, ardından yoğun bir rehabilitasyon gerektiren karmaşık bir ameliyattır. Kaynamama riski[ ${ }^{[31]}(\% 4,4-19)$ ve total diz replasmanı gibi başka bir cerrahiye gereksinim (\%5-40) riski barındırır. ${ }^{[32]}$ Olası riskler, komplikasyonlar ve endikasyonlar, hasta ile ayrıntılı olarak tartışılmalıdır. 


\section{KAYNAKLAR}

1. Lobenhoffer P. Indication for unicompartmental knee replacement versus osteotomy around the knee. J Knee Surg 2017;30(8):769-73. Crossref

2. Rossi R, Bonasia DE, Amendola A. The role of high tibial osteotomy in the varus knee. J Am Acad Orthop Surg 2011;19(10):590-9. Crossref

3. Puddu G, Cipolla M, Cerullo G, Franco V, Gianni E. Which osteotomy for a valgus knee? Int Orthop 2010;34(2):239-47. Crossref

4. Marti RK, Verhagen RAW, Kerkhoffs G, Moojen TM. Proximal Tibial Varus Osteotomy. J Bone Joint Surg Am 2001;83(2):16470. Crossref

5. Dejour D, La Barbera G, Pasqualotto S, Valoroso M, Nover L, Reynolds R, Saffarini M. Sagittal plane corrections around the knee. J Knee Surg 2017;30(8):736-45. Crossref

6. Zampogna B, Vasta S, Papalia R. Patient Evaluation and Indications for Osteotomy Around the Knee. Clin Sports Med 2019;38(3):305-15. Crossref

7. McNamara I, Birmingham TB, Fowler PJ, Giffin JR. High tibial osteotomy: evolution of research and clinical application-a Canadian experience. Knee Surg Sports Traumatol Arthrosc 2013;21(1):23-31. Crossref

8. Herman BV, Giffin JR. High tibial osteotomy in the ACLdeficient knee with medial compartment osteoarthritis. J Orthop Traumatol 2016;17(3):277-85. Crossref

9. Naudie DDR, Amendola A, Fowler PJ. Opening wedge high tibial osteotomy for symptomatic hyperextension-varus thrust. Am J Sports Med 2004;32(1):60-70. Crossref

10. Agneskirchner JD, Hurschler C, Stukenborg-Colsman C, Imhoff $A B$, Lobenhoffer P. Effect of high tibial flexion osteotomy on cartilage pressure and joint kinematics: a biomechanical study in human cadaveric knees. Winner of the AGA-Donjoy Award 2004. Arch Orthop Trauma Surg 2004;124(9):575-84. Crossref

11. Frank RM, Cotter EJ, Strauss EJ, Gomoll AH, Cole BJ. The utility of biologics, osteotomy, and cartilage restoration in the knee. J Am Acad Orthop Surg 2018;26(1):e11-25. Crossref

12. Rand J, Neyret P. ISAKOS meeting on the management of osteoarthritis of the knee prior to total knee arthroplasty. In: ISAKOS congress, 2005; Hollywood, Florida, USA.

13. Niemeyer $P$, Albrecht $D$, Andereya $S$, Angele $P$, Ateschrang A, Aurich M, Baumann M, Bosch U, Erggelet C, Fickert S, Gebhard H, Gelse K, Günther D, Hoburg A, Kasten P, Kolombe T, Madry H, Marlovits S, Meenen N, Müller P, Nöth U, Petersen J, Pietschmann M, Richter W, Rolauffs B, Rhunau K, Schewe B, Steinert A, Steinwachs M, Welsch G, Zinser W, Fritz J. Autologous chondrocyte implantation $(\mathrm{ACl})$ for cartilage defects of the knee: a guideline by the working group "clinical tissue regeneration" of the German Society of Orthopaedics and Trauma (DGOU). Knee 2016;23(3):426-35. Crossref

14. Sabzevari S, Ebrahimpour A, Roudi MK, Kachooei AR. High tibial osteotomy: A systematic review and current concept. Arch Bone Jt Surg 2016;4(3):204-12.

15. Bonasia DE, Dettoni F, Sito G, Blonna D, Marmotti A, Bruzzone M, Castoldi F, Rossi R. Medial openning wedge high tibial osteotomy for medial compartment overload/ arthritis in the varus knee: prognostic factors. Am J Sports Med 2014;42(3):690-8. Crossref

16. Dettoni F, Bonasia DE, Castoldi F, Bruzzone M, Blonna D, Rossi R. High tibial osteotomy versus unicompartmental knee arthroplasty for medial compartment arthrosis of the knee: a review of the literature. lowa Orthop |2010;30:131-40. https://www.ncbi.nlm.nih.gov/pmc/articles/PMC2958284/ pdf/iowa0030-0131.pdf
17. Flecher X, Parratte s, Aubaniac JM, Argenson JNA. A 12-28year follow up study of closing wedge high tibial osteotomy. Clin Orthop Relat Res 2006;452:91-6. Crossref

18. Javidan P, Adamson GJ, Miller JR, Durand P, Dawson PA, Pink MM, Lee TQ. The effect of medial opening wedge proximal tibial osteotomy on patellofemoral contact. Am J Sports Med 2013;41(1):80-6. Crossref

19. Goshima PK, Sawaguchi T, Shigemoto K, Iwai S, Nakanishi A, Ueoka K. Patellofemoral Osteoarthritis Progression and Alignment Changes after Open-Wedge High Tibial Osteotomy Do Not Affect Clinical Outcomes at Mid-term Follow-up. Arthroscopy 2017;33(10):1832-9. Crossref

20. Sadek AF, Osman MK, Laklok MA. Management of combined knee medial compartmental and patellofemoral osteoarthritis with lateral closing wedge osteotomy with anterior translation of the distal tibial fragment: Does the degree of anteriorization affect the functional outcome and posterior tibial slope. Knee 2016;23(5):857-61. Crossref

21. Lobenhoffer $P$, Agneskirchner JD. Improvements in surgical technique of valgus high tibial osteotomy. Knee Surg Sports Traumatol Arthrosc 2003;11(3):132-8. Crossref

22. Hinterwimmer S, Beitzel K, Paul J, Kirchhoff C, Sauerschnig $M$, von Eisenhart-Rothe R, Imhoff $A B$. Control of posterior tibial slope and patellar height in open-wedge valgus high tibial osteotomy. Am J Sports Med 2011;39(4):851-6. Crossref

23. El-Azab H, Glabgly $P$, Paul J, Imhoff AB, Hinterwimmer S. Patellar height and posterior tibial slope after open-and closed-wedge high tibial osteotomy: a radiological study on 100 patients. Am J Sports Med 2010;38(2):323-9. Crossref

24. LaPrade RF, Barrera Oro F, Ziegler CG, Wijdicks CA, Walsh MP. Patellar height and tibial slope after opening-wedge proximal tibial osteotomy: a prospective study. Am J Sports Med 2010;38(1):160-70. Crossref

25. Brinkman JM, Lobenhoffer P, Agneskirchner JD, Staubli AE, Wymenga $A B$, van Heerwaarden RJ. Osteotomies around the knee: patient selection, stability of fixation and bone healing in high tibial osteotomies. J Bone Joint Surg $\mathrm{Br}$ 2008;90$\mathrm{B}(12): 1548-57$. Crossref

26. Krych AJ, Hevesi M, Desai VS, Camp CL, Stuart MJ, Saris DBF. Learning fromfailure in cartilage repair surgery: an analysis of the mode of failure of primary procedures in consecutive cases at a tertiary referral center. Orthop J Sports Med 2018;6(5):2325967118773041. Crossref

27. Bode G, Schmal H, Pestka JM, Ogon P, Sudkamp NP, Niemeyer P. A non-randomized controlled clinical trial on autologous chondrocyte implantation $(\mathrm{ACl})$ in cartilage defects of the medial femoral condyle with or without high tibial osteotomy in patients with varus deformity of less than 5 degrees. Arch Orthop Trauma Surg 2013;133(1):43-9. Crossref

28. Takeuchi R, Ishikawa H, Miyasaka Y, Sasaki Y, Kuniya T, Tsukahara S. A novel closed-wedge high tibial osteotomy procedure to treat osteoarthritis of the knee: Hybrid technique and rehabilitation measures. Arthrosc Tech 2014;3(4):e431-7. Crossref

29. Uquillas C, Rossy W, Nathasingh CK, Strauss E, Jazrawi L, Gonzalez-Lomas G. Osteotomies About the Knee. J Bone Joint Surg Am 2014;96(24):e199(1-8).

30. Dejour D, Bonin N, Locatelli E. Tibial antirecurvatum osteotomies. Oper Tech Sports Med 2000;8(1):67-70. Crossref

31. Spahn G. Complications in high tibial (medial opening wedge) osteotomy. Arch Orthop Trauma Surg 2004;124(10):64953. Crossref

32. Amendola A, Bonasia DE. Results of high tibial osteotomy: review of the literature. Int Orthop 2010;34(2):155-60. Crossref 